Running Head: DEVALUATION OF ACCESSORY ITEMS

Affective evidence that inhibition is involved in separating accessory representations

from the active representation in visual working memory

David De Vito and Mark J. Fenske

Department of Psychology, University of Guelph, Ontario Canada

Words in abstract: 149

Words in main text body: 7,496

Number of references: 54

Author Information (*corresponding author):

*Mark J. Fenske, PhD

Department of Psychology

University of Guelph

Guelph, Ontario N1G 2W1

Email: mfenske@uoguelph.ca

Tel: 519-824-4120 x56411

Fax: 519-837-8629

David De Vito, MSc

Department of Psychology

University of Guelph

Guelph, Ontario N1G 2W1

Email: ddevito@uoguelph.ca

Tel: 226-971-3814 


\title{
Affective evidence that inhibition is involved in separating accessory representations from the active representation in visual working memory
}

\begin{abstract}
The multiple state theory of working memory suggests that representations are divided into two states: a focused-on active representation and accessory memories held for later use. Here, we tested two competing hypotheses regarding the neurocognitive mechanisms responsible for this separation: (1) that accessory memories undergo inhibition or (2) that accessory memories are amplified less than active representations. We explored if accessory memories undergo affective devaluation, a known index of the involvement of inhibition in a visual task. On each trial participants memorized four items, were cued to focus on one, and then completed a visual search or an affective evaluation task. While search distractors matching the colour of an active item slowed search, those matching an accessory memory did not, replicating previous findings that only active items guide search. Also, accessory items were affectively devalued compared to baseline and active items, supporting the hypothesis that accessory memories undergo inhibition.
\end{abstract}

Key words: working memory, affective evaluation, devaluation-by-inhibition, accessory memory 


\section{Introduction}

Trying to locate your car in a crowded parking lot is a situation that many people experience quite frequently. Success in this task is dependent on the ability to direct attention towards your car (i.e., the task-relevant stimulus) while preventing distraction from task-irrelevant items. And the ability to quickly find such a target object is aided by maintaining a representation of that item in visual working memory, which acts as an attentional template to help guide search (Carlisle \& Woodman, 2011; Wolfe, 1994). According to the biased competition model, maintaining a target representation in working memory aids search by pre-activating neurons in visual cortex that code for the features associated with the target (Desimone \& Duncan, 1995). This pre-activation of neurons biases attentional processing in favour of external sensory items that share features with the attentional template, causing attention to be drawn towards (i.e., captured by) memory-matching items. It makes sense that this kind of match between the contents of working memory and information in the external world would be helpful for doing something like finding your car.

But what happens when the information being held in working memory is not immediately relevant to the task at hand, but must nevertheless be maintained for some later use? Is attention still biased toward external sensory stimuli that match the features of this kind of working memory representation? Perhaps not. Recent findings suggest that representations in working memory are maintained in one of two different states depending on their current relevance (Olivers, Peters, Houtkamp, \& Roelfsema, 2011). While immediately-relevant representations, such as an attentional target template, are placed in an 'active' state, representations that are not immediately relevant but are being 
preserved for later use are placed in an 'accessory' state. Importantly, the likelihood that selective attention is drawn to external stimuli that match the contents of working memory appears to be much greater for active representations than for accessory representations (Houtkamp \& Roelfsema, 2006), which thereby limits the interference that might otherwise result if attention were drawn to stimuli that match currentlyirrelevant working memory representations (Downing \& Dodds, 2004; Peters, Goebel, \& Roelfsema, 2009; Woodman \& Luck, 2007). While evidence regarding the existence and basic characteristics of these different memory states continues to grow (Eimer, 2015; Olivers et al., 2011; van Moorselaar, Theeuwes, \& Olivers, 2014), the exact neurocognitive mechanisms that are used to separate the accessory representations from the active representations working-memory remain unclear.

One possibility regarding the separation of accessory representations from the active item in working memory is that inhibition is applied when an item is determined to be currently irrelevant to reduce its activity in the sensory regions that code for its specific features (Peters, Roelfsema, \& Goebel, 2012). While the accessory representations would still be maintained in working memory so that they could be used later on, and would therefore maintain a level of activation above baseline, inhibition would be used to reduce their activity level below that of the active item. Other accounts, however, do not posit a role for inhibition, per se, but instead characterize the difference between accessory and active states as being about differences in magnitude of cortical amplification that increase from baseline levels (Lewis-Peacock, Drysdale, Oberauer, \& Postle, 2012) or from a relatively smaller subset of neurons (Kornblith, Quian Quiroga, Koch, Fried, \& Mormann, 2017) to maximal levels across a much larger number of 
neurons, respectively. Therefore, while both hypotheses suggest that accessory representations would maintain a certain level of activation because they are being held for a later task, they differ regarding their proposed involvement of inhibition. To test these competing accounts we sought evidence of the possible involvement of inhibition in separating accessory working memory representations from the active representation. Specifically, we examined whether a marker of inhibition — the distinctly negative affective evaluations that result from the inhibition of sensory stimuli (Raymond, Fenske, \& Tavassoli, 2003), their representations in memory (De Vito \& Fenske, 2017; Vivas, Marful, Panagiotidou, \& Bajo, 2016) or associated motor-responses (Fenske, Raymond, Kessler, Westoby, \& Tipper, 2005; Kiss, Raymond, Westoby, Nobre, \& Eimer, 2008)— would be evident in the stimulus ratings of items that had just been maintained in visual working-memory in an accessory state. Finding affective devaluation of prior accessory items, relative to baseline and prior active items, would be consistent with the view that inhibition is used to reduce the activation of currently-irrelevant working-memory items in an accessory state below the activation level of the active memory representation. In contrast, positive affective evaluations can be indicative of increasing levels of active stimulus representations (Craver-Lemley \& Bornstein, 2006; Kirby \& Greenwald, 2017; Nunoi \& Yoshikawa, 2016; Yagi, Ikoma, \& Kikuchi, 2009). Therefore, finding similar or more positive affective ratings for prior accessory items than baseline items would not be consistent with an inhibition account of the difference between accessory and active states, but would instead be consistent with an account based on differences in cortical amplification of the corresponding neural representations. 


\section{Inhibition leads to Stimulus Devaluation}

The evidence that inhibition is linked to the affective devaluation of associated visual stimuli consists of the typical finding that items that appear in experimental conditions thought to require inhibition for successful task performance subsequently receive more negative affective ratings than previously-unseen baseline items or items from conditions thought to involve no inhibition (Fenske \& Raymond, 2006; Gollwitzer, Martiny-Huenger, \& Oettingen, 2014; Raymond, 2009). Such evidence has accumulated across a variety of attention tasks that require participants to avoid interference from distractors, including those involving two-item target localization (Raymond et al., 2003), simple-feature and difficult-conjunction search (Raymond, Fenske, \& Westoby, 2005), preview search (Fenske, Raymond, \& Kunar, 2004), and central-target discriminations (Martiny-Huenger, Gollwitzer, \& Oettingen, 2014). Affective devaluation also occurs for stimuli from which a motor-response has been withheld in Go/No-go (Kiss et al., 2008) and Stop-signal (Wessel \& O’Doherty, 2014) tasks, and whenever a mental representation of the item is ignored in working memory (De Vito, Ferrey, Fenske, \& AlAidroos, 2018) or is suppressed in long-term memory during Think/No-Think (De Vito \& Fenske, 2017) or item-based directed-forgetting (Vivas et al., 2016) tasks.

\section{Stimulus Devaluation as an Index of Inhibition}

The consistency of the link between inhibition and subsequent negative stimulus ratings has prompted some researchers to begin using stimulus devaluation as a critical indicator of the potential involvement of inhibition in a given visual task (Fenske et al., 2004; Kihara, Yagi, Takeda, \& Kawahara, 2011). Kihara et al. (2011), for example, used stimulus evaluations to resolve uncertainty about whether the attentional blink- the 
impairment in identifying the second of two visual targets when it follows within $500 \mathrm{~ms}$ of the first (Raymond, Shapiro, \& Arnell, 1992) — is a result of inhibition. According to the inhibition account, the deficit in target processing is caused by inhibition triggered by an intervening distractor that not only reduces interference from the distractor but also inadvertently impacts other processing for a brief period thereafter (Olivers \& Meeter, 2008). Kihara et al. found that intervening distractors from trials showing the attentional blink effect were affectively devalued, as would be expected if they had been inhibited. The authors took this close link between the affective marker of inhibition and the occurrence of the attentional blink as strong support for the inhibition account over other competing explanations.

Fenske et al. (2004) found that the occurrence of distractor devaluation was predictive of another attention-related effect posited to involve distractor inhibition- the preview benefit in visual search (Watson \& Humphreys, 1997; 2000). In the critical preview trials of this task, a subset of distractors is presented for a brief period before the search target and the remaining distractors. The benefit of this preview is faster localization of the target on preview trials than on non-preview trials (in which all distractors appear simultaneously with the target). Affective ratings obtained by Fenske et al. after search trials revealed that previewed distractors are devalued relative to nonpreviewed distractors, but this only occurs for participants who show the preview benefit. This close link between the occurrence of the preview benefit and the affective marker of inhibition was taken by the authors as converging support for the distractor-inhibition account of the preview benefit over other competing non-inhibition accounts. 
By using stimulus devaluation as an index of the potential involvement of inhibition within a visual task, the experimental approach taken here is therefore similar to that of both Kihara et al. (2011) and Fenske et al. (2004).

\section{Positive Affect as an Index of Active Stimulus Representations}

Just as negative affective evaluations can be indicative of attention-, memory-, and response-related inhibition, positive affective evaluations can be indicative of increasing activation of stimulus representations (Craver-Lemley \& Bornstein, 2006; Kirby \& Greenwald, 2017; Nunoi \& Yoshikawa, 2016; Yagi et al., 2009). Stimulus repetition, for example, is a well-established method of raising the activation level of the cortical representations of visual objects, which acts to facilitate their processing in subsequent encounters (Henson \& Rugg, 2003; Schacter \& Buckner, 1998; Vorberg, Mattler, Heinecke, Schmidt, \& Schwarzbach, 2003; Zago, Fenske, Aminoff, \& Bar, 2005). The mere exposure effect - the increase in stimulus liking that accompanies increases in stimulus repetition (Zajonc, 1968; 2001) — is therefore a classic example of the link between the amplification of stimulus representations and positive affect. More recent work suggests further that increases in the depth of processing and extent to which a stimulus is actively brought to mind and maintained in working memory is also accompanied by corresponding increases in positive evaluations of such items (CraverLemley \& Bornstein, 2006; Inoue, Yagi, \& Sato, 2018; Kirby \& Greenwald, 2017; Nunoi \& Yoshikawa, 2016).

In Kirby \& Greenwald's (2017) investigation, for instance, stimuli that participants were asked to mentally rehearse while completing other cognitive tasks subsequently received more positive ratings than stimuli that were not held in memory, 
but were otherwise comparable in terms of stimulus exposure and other critical factors. Furthermore, this effect of memory maintenance on increasingly positive stimulus evaluations was robust across a variety of stimulus types and intervening tasks. A clear prediction from these results is that the amplified cortical response needed to represent stimuli in an active state in visual working memory should result in significantly more positive affective evaluations of these items than baseline items or prior accessory-state items. But what about accessory items relative to baseline? If an accessory state is achieved through cortical representations that are maintained at a baseline level of activation (Lewis-Peacock et al., 2012) or are amplified in some neurons but not a sufficiently large number to be in an active state (Kornblith et al., 2017), then ratings of prior accessory items should be similar to or slightly more positive than those of baseline items, but not as positive as prior active items. Thus, in terms of affective evidence that might distinguish between an inhibition-based account and a levels-of-amplification account, the critical question is whether affective ratings of prior accessory items are more negative or similar / more positive than baseline items.

\section{Testing the Competing Hypotheses}

To test the competing predictions that visual working-memory representations in an accessory state are associated with more negative evaluations (inhibition hypothesis) or similar / more positive evaluations (levels-of-amplification hypothesis), we integrated a stimulus rating procedure within a working-memory paradigm that utilizes retro-cues to manipulate whether visual stimuli are maintained in an active or accessory state (van Moorselaar, Theeuwes, \& Olivers, 2014; van Moorselaar, Olivers, Theeuwes, Lamme, \& Sligte, 2015). On each trial of our experiment, participants memorized four meaningless 
visual patterns. After the array of to-be-memorized items disappeared, a retro-cue appeared indicating which of the memorized items should become the active focus of an upcoming memory test (i.e., is this the cued item?) and which should be reserved for a second memory test later on. van Moorselaar et al. $(2014 ; 2015)$ established that the immediate relevance of such retro-cued items for the upcoming memory probe caused them to become represented in an active state, whereas the remaining non-cued items were maintained in an accessory state. To verify the effectiveness of this procedure for creating active vs. accessory working-memory states, we included a subset of trials for one group of our participants (i.e., the Search group) in which a retro-cue was immediately followed, not by a memory probe as expected, but rather a visual search task in which one of the distractors matched the colour of the cued item (i.e., the active item), one of the uncued items (i.e., an accessory item), or was a novel colour (i.e., baseline). If our modified version of van Moorselaar et al.'s (2014) retro-cue procedure is effective at manipulating whether working memory representations are maintained in active versus accessory states, then we expect the results of these visual search trials to replicate their past findings that a memory-matching distractor captures attention and significantly slows down target localization only when the distractor-matching working-memory representation is in an active state and not when it is in an accessory state. This is exactly what we found.

All of our participants (i.e., the Search group and No Search group) completed trials that integrated stimulus ratings (How cheerful is this item?) within workingmemory retro-cue trials. For the critical experimental trials, the rating display occurred immediately after the appearance of a retro-cue that indicated which of the four items 
from the initial visual array was to be the active focus for the impending memory test, and which were to be reserved for a second memory test later on. The to-be-rated stimulus on each of these trials was either the cued (active-state) item or one of the uncued (accessory-state) items. To provide a proper baseline against which these accessory-item and active-item ratings could be compared, we intermixed the critical trials with baseline trials in which the stimulus-rating display appeared immediately after the initial visual array, prior to the appearance of a retro-cue. While little is known about the exact point at which working-memory representations are differentiated into active and accessory states, existing evidence suggests that it does not happen until information about their relative priority becomes available; for instance, upon the presentation of a retro-cue. van Moorselaar and colleagues $(2014 ; 2015)$, for example, have demonstrated that when multiple items of equal relevance are maintained in visual working memory, none of the representations guided attention unless a retro-cue appeared to indicate which should receive priority. Therefore, in our baseline condition, in which participants have encoded four items into working memory, but have not yet been presented with any cues to indicate relative priority, all four working-memory representations should have equal relevance and not yet be differentiated into active or accessory states. The appropriateness of the ratings of these items as a baseline for assessing the affective impact of the processes that differentiate working-memory representations into active and accessory states therefore concerns the fact that our baseline ratings are evaluations of items from the same stimulus set as the active and accessory items that are matched in level of sensory exposure, but have neither been fully set into an active state in working memory nor into an accessory state. 
The extent to which our affective-rating results can help to distinguish between the inhibition-based account and a levels-of-amplification account of how visual stimuli are represented in different working-memory states, primarily concerns whether evaluations of prior accessory items are found to be more negative or similar to (or more positive) than those of baseline items. To preview our results, ratings of prior accessory items were more negative than those for baseline items and prior active items in both groups of participants.

\section{Method}

Participants. Fifty-two undergraduate students were recruited using the participant pool at the University of Guelph and participated in the experiment in exchange for course credit. Twenty-four participants were randomly assigned to the No Search Group, while the remaining twenty-eight participants were assigned to the Search Group. All of the participants provided informed consent and all had normal or correctedto-normal vision. Three participants were removed from the data analysis because they scored an overall accuracy below chance (i.e., 25\%). This resulted in a final sample size of N=49 participants (Age: $M=19.1$ years, $S D=1.95 ; 17$ females, 23 right-handed) combined across both groups. The size of this total sample was consistent with the samples of 40-50 participants used in previous investigations of the affective consequences of inhibition (e.g., Fenske et al., 2004; Ferrey, Frischen, \& Fenske, 2012; Raymond et al., 2005). The appropriateness of this sample size was confirmed using G*Power (Faul, Erdfelder, Lang, \& Buchner, 2007), which indicated that 45 participants would be needed to detect a similar-sized effect on stimulus ratings as in these studies $\left(d_{z}\right.$ 
$=.5$ ). The sample size of the subset of 26 participants that comprised the Search Group was also chosen to be consistent with the samples of 15-25 participants used in previous investigations of attentional capture by memory-matching distractors (van Moorselaar et al., 2014; 2015).

Apparatus \& Stimuli. The stimuli used for this experiment were the same as those used in De Vito et al. (2018, Experiment 2) in our demonstration that stimuli whose representations are ignored in visual working memory become affective devalued. The Mondrian stimuli $\left(1.45^{\circ} \times 1.45^{\circ}\right)$ were created using a script in E-Prime (Psychology Software Tools, Pittsburgh, PA) that randomly generated 200 overlapping squares (each between $10-30 \%$ of the width of the full stimulus) within a larger square border. All of the overlapping squares in each stimulus were originally drawn to be the exact same colour (Blue, Green, Orange, Pink, Purple, or Yellow). Later, Adobe Photoshop was used to make the overlapping squares within each stimulus varying degrees of the main initial colour. Five-hundred stimuli of each of the main colours were used, for a total of threethousand stimuli. For the No Search Group, only the Blue, Green, Purple, and Yellow stimuli were used. For the Search Group we replaced the Yellow stimuli with Orange to improve singleton salience, and also needed an additional fifth colour to incorporate novel singletons so we added Pink stimuli. All stimulus presentation and behavioral response collection for this experiment were controlled using PsychoPy software (Peirce, 2007) running on a personal computer with a $50.8 \mathrm{~cm}$ LCD monitor (resolution: $1920 \mathrm{x}$ 1080 pixels). Participants sat at a viewing distance of approximately $71 \mathrm{~cm}$ from the monitor.

\section{Experimental Procedure}


All of the materials and procedures used in this experiment were approved by the Research Ethics Board at the University of Guelph.

All participants completed experimental trials that combined a retro-cue workingmemory task and an affective-evaluation task. There were two types of these Memory + Affective-evaluation trials that differed only in the point at which the Affectiveevaluation display was presented to obtain stimulus ratings. In the Critical trials, the Affective-evaluation task occurred immediately after a retro cue appeared to induce justmemorized stimulus representations to be in active or accessory states. In Baseline trials, the Affective-evaluation task occurred before the retro-cue and thus enabled ratings to be obtained of just-memorized stimuli before their corresponding working-memory representations could be fully set into active or accessory states. In addition to the Memory + Affective-evaluation trials, participants in the Search group completed trials that combined a retro-cue working-memory task with a visual-search task. The No Search group participants completed a total of 128 trials, comprised solely of Memory + Affective-evaluation trials (64 critical trials; 64 baseline trials). The Search group participants completed a total of 136 trials, comprised of 64 Memory + Affectiveevaluation trials (32 critical trials; 32 baseline trials) and 72 Visual-search trials.

\section{Baseline Trials}

Each new trial began with a $500 \mathrm{~ms}$ presentation of the word "Memorize" at display center, followed by a $250 \mathrm{~ms}$ central fixation cross, and the appearance of the memory array. Each memory array consisted of four stimuli located $2.42^{\circ}$ away from fixation in one of the four corners of an imaginary square (see Figure 1). Stimuli used for each array were randomly selected with the constraint that the main colour was different 
for each of the four items. A staircase procedure was used to increase or decrease the presentation duration of the memory array based on the recent accuracy of the participant's memory-test responses. At the beginning of the experiment the to-bememorized items were presented for $500 \mathrm{~ms}$. Accuracy was calculated following each trial. Trials where the participant responded correctly to both probe stimuli were classified as correct, while responding correctly to only one or neither of the probe stimuli meant that the trial was classified as incorrect. When accuracy over the four previous trials exceeded $75 \%$, the presentation duration of the memory array decreased by $50 \mathrm{~ms}$; when accuracy fell below $75 \%$, the duration increased by $50 \mathrm{~ms}$. The only constraint to this staircase procedure was that memory-array display duration always remained between 150-1000 ms. Following the disappearance of the memory array, there was an 800 ms retention interval during which only the central fixation cross was visible. This was followed by the Affective-evaluation task.

The Affective-evaluation task began with a 500 ms presentation of the word "Evaluate" at display center to alert participants that an evaluation task was starting. A centrally-presented to-be-rated stimulus was then presented with the numbers 1-2-3-4 centered horizontally $2.82^{\circ}$ below fixation for $1000 \mathrm{~ms}$ followed by a blank screen. Participants were asked to evaluate the to-be-rated stimulus based on its cheerfulness from 1 (not at all cheerful) to 4 (very cheerful) using the number keys in the top left corner of the keyboard. The rest of the trial—preceded by a $250 \mathrm{~ms}$ central-fixation display — only resumed once the participant had made a response.

The first retro-cue followed immediately after the Affective-evaluation task. The retro-cue was a $200 \mathrm{~ms}$ central arrow that pointed to the location of one of the four 
memorized items (pointed-to location counterbalanced across trials). Its appearance alerted participants to remember the item that had appeared in that location for an upcoming memory test. The retro-cue was $100 \%$ valid in that the cued item was always the focus of the memory test. The disappearance of the first retro-cue was immediately followed by another $800 \mathrm{~ms}$ retention interval.

The memory test regarding the item indicated by the first retro-cue followed immediately after the retention interval, and began with a $500 \mathrm{~ms}$ appearance of the word "Same?" at display center to alert the participants of the upcoming memory probe. A 250 ms central-fixation display separated this alert display and the subsequent $1000 \mathrm{~ms}$ appearance of the memory probe at display center. As the purpose of the memory probe was to ensure that participants had good memory for the memory array, while maintaining a high level of difficulty, all four items from the memory array could appear as the memory probe stimulus. On $50 \%$ of the trials the memory probe was the cued stimulus from the memory array, while on the other $50 \%$ of the trials, the probe was one of the three uncued stimuli from the memory array. Using the up and down arrow keys, participants pressed one key to indicate if the probe matched the cued stimulus and the other if it did not match (key-to-response assignment counterbalanced across participants). Participants were able to respond at any point during the presentation of the memory-probe item or the blank display that followed its disappearance. The rest of the trial, which consisted of a second retro-cue and memory-probe sequence, only proceeded once the participant had made a memory-test response.

As we were interested in the differences in affective evaluations of items held in different states of working memory, it was crucial that participants maintained all items 
in working memory through the conclusion of the evaluation task. If items became irrelevant after the first retro-cue then participants would have no motivation to maintain items in accessory memory. To confirm that participants were indeed maintaining items in memory following the first retro-cue, each memory trial contained a second retro-cue and second memory probe that cued participants to an item in memory and tested its identity, respectively. The second retro-cue was preceded by an $800 \mathrm{~ms}$ central-fixation display and was otherwise similar in all respects to the first retro-cue, except that the location it pointed to was contingent on which location had been pointed to by the first retro-cue. Specifically, the second retro-cue only ever pointed to one of the two memoryarray locations in the opposite hemifield as that of the location indicated by the first retrocue (i.e., if the first retro-cue had pointed to a location on the left-hand side of the display, then the second retro-cue would point to one of the two locations on the righthand side). As always, the appearance of the retro-cue alerted participants to remember the item that had appeared in the cued location for an upcoming memory test. The timing and sequence of events for the second memory test was exactly the same as that for the first memory test (i.e., $800 \mathrm{~ms}$ retention interval, then $500 \mathrm{~ms}$ 'Same?' alert, then $250 \mathrm{~ms}$ central-fixation, then $1000 \mathrm{~ms}$ memory-probe, then a blank display until the memory-test response was provided). Similar to the first memory probe, any of the four memory items could appear as the second memory probe to maintain a high level of difficulty on the memory test. The second probe was the just-cued item from the memory array on $50 \%$ of trials, and one of three remaining (not-just-cued) items on the other $50 \%$ of trials.

\section{Critical Trials}


The Critical and Baseline versions of the Memory + Affective-evaluation trials had the exact same timing and sequence of events, aside from the point at which the Affective-evaluation task occurred. This meant that the Critical Trials differed from the Baseline Trials in the following ways. On Critical Trials, the first retro-cue occurred immediately following the first retention interval. Following the second retention interval, that which followed the disappearance of the first retro-cue, was when the Affective-evaluation task occurred in which the cued item (i.e, the active item) or one of the uncued items (i.e., one of the accessory items) was presented as the to-be-rated stimulus. When an uncued item was selected to appear as the to-be-rated stimulus, it was always one of the items from the hemifield opposite to that of the cued location. The first memory test immediately followed the Affective-evaluation task. Finally, the item pointed to by the second retro-cue on Critical trials was never an uncued item presented as the to-be-rated stimulus during the immediately preceding Affective-evaluation task.

The exact duration of each event and number of trials from each condition is shown separately in Table 1 for the No Search and Search groups. In general, both groups had the same timing and sequence of stimulus events for the Critical and Baseline trials, with the exception that the text alerts for each task were presented for $800 \mathrm{~ms}$ for the Search group (vs. $500 \mathrm{~ms}$ for No Search participants), and the memory-retention intervals were each $1000 \mathrm{~ms}$ in duration for the Search group (vs. $800 \mathrm{~ms}$ for No Search participants).

\section{Visual Search Task}

The Visual-search trials were randomly intermixed with the Memory + Affectiveevaluation trials for participants in the Search group. Each began with the same memory- 
array, retention interval and retro-cue sequence of events that occurred at the start of each of the Critical trials, including the retention interval following that first retro-cue. In the Visual-search trials, however, participants were presented at this point with a search array consisting of eight Landolt C stimuli (Woodman \& Luck, 2007), rather than the Affective-evaluation display of the Critical trials. The appearance of the search array alerted participants that their task was to now find the single target item in the array and indicate as quickly and accurately as possible whether the gap in the target stimulus was at its top or bottom (all other items had a gap on the left or right). They were instructed to respond with the up-arrow key to indicate that the gap was at the top of the Landolt $\mathrm{C}$ stimulus or the down-arrow key to indicate that the gap was at the bottom. The eight stimuli in the search array were positioned around central fixation at equal intervals along a circular pattern, the center of each $2.02^{\circ}$ from display center. The eight items included 1 target, 6 regular distractors, and 1 colour-singleton distractor. The target and distractors were always grey in colour. The colour of the singleton was novel, the same as the cued item, or the same as one of the uncued stimuli in the hemifield opposite to that of the cued location. The search array remained on the screen until the participant made a response.

Prior to the experimental trials, participants completed 20 practice trials that otherwise exactly matched the experimental trials. For the No Search group, these practice trials included 10 Baseline trials and 10 Critical trials. For the Search group, the practice trials included 7 Baseline trials, 7 Critical trials, and 6 Visual-search trials. Participants received feedback after each response during the practice trials in the form of the word "Correct" or "Incorrect" displayed in the center of the screen for $150 \mathrm{~ms}$. 


\section{Results \& Discussion}

Participants responded accurately to the first memory probe on $84.8 \%(S D=9.3)$ of trials and to the second memory probe on $83.8 \%(S D=10.8)$ of trials. As this investigation examined different states of representations in working memory it was critical that participants be able to maintain all representations through the length of each trial. Finding that participants responded correctly to both the first and second memory probe on $63.6 \%(S D=17.9)$ of trials, well above chance level (i.e., $25 \%)$, confirmed that our staircase method of varying array presentation time was successful in maintaining a consistently high level of difficulty, while also ensuring that participants were able to maintain the memorized stimuli throughout the length of the trial. Participants responded correctly to both the first and second memory probes more frequently during baseline trials $(M=66.2 \%, S D=2.0)$ than on critical trials $(M=61.1 \%, S D=1.8), t(48)=2.93, p$ $=.005$. This result suggests that presenting the evaluation task following the retro-cue did potentially interfere with memory encoding. While this result was not applicable to our particular hypotheses, it may inform future research that combines retro-cue tasks with secondary tasks (e.g., affective evaluation tasks).

We therefore proceeded with our investigation of the affective consequences associated with manipulating the state of items in working memory. Our investigation was particularly interested in trials where participants maintained the memory items for the length of the trial, and errors are known to be associated with affective consequences (Chetverikov, Jóhannesson, \& Kristjánsson, 2015; Chetverikov, 2014) so we restricted our analysis of affective evaluations to trials where the participant was correct in their 
responses to both the first and second memory probes. This resulted in the removal of the ratings of $30 \%$ of Baseline items, $38 \%$ of Accessory Items, and 35\% of Active Items. The affective ratings were entered into a 2(Group: No Search vs. Search) x 3(Stimulus Type: Baseline vs. Accessory Item vs. Active Item) mixed ANOVA. The results of the ANOVA showed a main effect of Stimulus Type, $F(2,94)=16.64, p<.001, \eta_{\text {partial }}^{2}=.261$. There was no main effect of Group, $F(1,47)=.16, p=.693, \eta^{2}$ partial $=.003$, and no Stimulus Type $\mathrm{x}$ Group interaction, $F(2,94)=1.30, p=.277, \eta_{\text {partial }}^{2}=.027$. The lack of significant interaction or main effect of Group suggests that the presence of search trials did not influence the prioritization of representations in working memory and therefore did not influence affective ratings. Consequently, we investigated the effect of Stimulus Type by combining the affective rating results from both Groups into one larger analysis. Our finding that participants rated active memory items $(M=2.95, S D=0.48)$ significantly higher than baseline items $(M=2.72, S D=0.37), t(48)=3.36, p=.002$, replicates previous work showing that increases in depth of processing such as actively maintaining an item in memory is accompanied by increased positive evaluations (Craver-Lemley \& Bornstein, 2006; Inoue et al., 2018; Kirby \& Greenwald, 2017; Nunoi \& Yoshikawa, 2016). Our novel finding here involves the affective ratings of accessory memory items. Paired-samples t-tests showed that participants rated accessory memory items $(M=2.51$, $S D=0.40)$ significantly lower than baseline items $(M=2.72, S D=0.37), t(48)=3.40, p$ $=.001$, and active memory items $(M=2.95, S D=0.48), t(48)=4.94, p<.001$. The finding that accessory memory items were rated more negatively than baseline items, as opposed to following a similar pattern as active items, specifically supports the inhibition hypothesis, rather than the levels of activation hypothesis. The devaluation of accessory 
items suggests that there are affective consequences associated with manipulating the states of representations in working memory, in particular with maintaining currently irrelevant representations for later use (i.e., in an accessory state). These findings of affective devaluation were not a product of varying stimulus exposure due to the staircase method, as a one-way ANOVA revealed that there was no significant difference in average encoding duration on trials where a Baseline $(M=0.83, S D=0.17)$, Accessory $(M=0.84, S D=0.19)$, or Active item $(M=0.83, S D=0.18)$ were evaluated, $F(2,96)=$ $1.231, p=.297$. Similar to previous investigations that have used stimulus devaluation as an index of the potential involvement of inhibition in a visual task (Fenske et al., 2004; Kihara et al., 2011), here we interpret the presence of affective devaluation to be an index of the involvement of inhibitory processes in separating representations in an accessory state in working memory from the active representation (Peters et al., 2012).

Participants responded accurately on the search task on $98.6 \%(S D=2.0)$ of trials. Removing incorrect trials from the analysis therefore resulted in the removal of $1.4 \%$ of total trials, and data trimming removed an additional $2.7 \%$ of trials. To confirm if our retro-cue procedure was effective at manipulating the states of representations in working memory we analyzed the attentional capture during visual search of the three kinds of singleton distractors (i.e., active-matching, accessory-matching, and novel). Response times on trials where the singleton matched the colour of the currently active memory item (i.e., active-matching; $M=1.33, S D=0.39$ ) were significantly slower than on trials where the singleton was a novel colour $(M=1.27, S D=0.32), t(26)=2.69, p=.012$. However, there was no difference in response times on trials where the singleton matched the colour of an accessory item (i.e., accessory-matching; $M=1.29, S D=0.34$ ) compared 
to trials when the singleton was a novel colour $t(26)=1.35, p=.188$. These findings suggest that our search task was effective in replicating past findings where only one item in memory can guide attention (Downing \& Dodds, 2004; van Moorselaar et al., 2014), as items matching a feature of the cued (i.e., active) item significantly slowed response times. Visually inspecting the average response times shown in Figure 3 clarifies that there was some nominal slowing of responses by accessory-matching singletons that did not reach significance. This finding aligns with findings of Houtkamp and Roelfsema (2006) who found that accessory items captured attention in search in the absence of the active item. While we did not find significant attentional capture by accessory-matching items in the absence of the active item, Houtkamp and Roelfsema presented the actual accessory memory item in the array, while here we only presented items that matched a feature of the accessory memory item (i.e., colour).

\section{General Discussion}

The multiple state account of working memory attempts to explain the mixed findings regarding whether attention is guided towards items that match features of the contents of working memory. The model hypothesizes that representations held in working memory are separated into two distinct states, with one representation being the focus of attention (i.e., active) and the remaining being held for future use (i.e., accessory; Olivers et al., 2011). The neurocognitive mechanisms underlying the apparent split between representations in working memory have only sparsely been investigated.

Our experiment explored the competing hypotheses that accessory memory items undergo inhibition to reduce their level of activation below that of the active item (Peters 
et al., 2012), or instead simply undergo less activation than active memory items (Kornblith et al., 2017; Lewis-Peacock et al., 2012). Therefore, the two hypotheses differ only in regards to their proposed involvement of inhibitory processes. Similar to Fenske et al. (2004) and Kihara et al. (2011), we used stimulus devaluation as an index of the presence of inhibitory processes.

On each trial of our experiment, participants memorized a four-item memory array and then were asked to evaluate one of the memorized items (i.e., evaluation trials) or to complete a visual search task (i.e., search trials). Our results showed that on search trials participants took longer to respond (i.e., their attention was captured) when a distractor matched a feature of the active memory item, compared to a novel-coloured distractor. However, when the singleton distractor matched a feature of the accessory memory item, significant attentional capture was not found. This capture by active memory items replicates previous findings of a division of states within working memory (Houtkamp \& Roelfsema, 2006; Olivers et al., 2011; van Moorselaar et al., 2014), whereby only one item in memory can guide attention. On evaluation trials, accessory memory items were significantly devalued compared to baseline items, suggesting that separating representations into different states in working memory has affective consequences for accessory items. Collectively, here we found that when a retro-cue is used to cue the focus of attention in working memory to a single representation, visual distractors matching features of the active memory item are more likely to capture attention than those matching features of accessory items, and accessory items also undergo an affective devaluation compared to baseline stimuli. 
Our previous research used a similar methodology paired with EEG to show that ignoring working memory representations has negative affective consequences (De Vito et al., 2018; Experiment 2). However, on each trial of our previous study participants were only presented with one retro-cue and one memory probe following the memory array. Therefore, participants had no use for the uncued items following the retro-cue and therefore no incentive to maintain the uncued items in working memory. Our results here, showing that participants were successful in responding to the second memory probe, suggest that they were able to successfully maintain the uncued items in working memory following the presentation of the first retro-cue. Our previous investigation could not differentiate whether the affective devaluation of uncued items compared to cued items was a result of representations being re-prioritized in working memory or from uncued items being removed from working memory completely. Here, we show that affective devaluation of uncued items results from re-prioritizing representations in working memory, while they are still being maintained in memory. If the first retro-cue had caused participants to delete all uncued memory representations from working memory then it would be expected that participants would have performed at chance-level accuracy when responding to the second memory probe, which was not the case.

Using a similar retro-cue and memory probe methodology as we used here, van Moorselaar et al. (2014) found that presenting a retro-cue between a memory array and a search task lead to attentional capture by search distractors that matched the cued item. Trials with no retro-cue showed no memory-driven capture. They suggested that presenting a retro-cue caused participants to move the cued representation into a prioritized active state, giving it the similar ability to capture attention as would be seen if 
only one single item was maintained in working memory. This suggestion implies that memorized items are immediately made accessory and then cued items are given a prioritized status. While the increased positive affect that we find to be associated with making a stimulus an active memory item does align with this hypothesis, our finding showing devaluation of accessory items does not. Our results suggest that the presentation of a retro-cue does not solely cause one item to be given a priority status, but causes items to be separated into a currently-relevant active item and accessory items held in the background for later use. The devaluation that we found here of accessory items following a retro-cue compared to baseline items suggests that along with prioritizing the cued item, a retro-cue causes uncued items to be inhibited and subsequently affectively devalued.

Traditionally, inhibition's involvement in visual search paradigms, such as preview search or search involving negative templates, has been to decrease the activation associated with irrelevant items and thereby facilitate search (Reeder, Olivers, \& Pollmann, 2017; Watson \& Humphreys, 1997; 2000). The key difference between these tasks and the role of inhibition in the differentiation of states in working memory is that accessory representations, which are possibly subject to inhibition, are needed later on. If inhibition's role in differentiating states in working memory is to facilitate search then it would be expected that search would be facilitated on trials that contained an accessory-matching singleton. However, our findings of similar search times on trials with an accessory-matching singleton compared to those with a novel singleton match the findings of many past investigations regarding the different states in working memory (van Moorselaar et al., 2015; Dirk van Moorselaar, Theeuwes, \& Olivers, 2016; c.f., 
Downing \& Dodds, 2004). Collectively, these findings suggest that the role of inhibition during the differentiation of states, rather than being to facilitate search, may instead be to govern (but not eliminate) the activation of accessory items to stay at no more than baseline levels to ensure that they can not guide attention but can easily be fully reactivated when needed for a task later on. These findings help to explain those of LewisPeacock et al. (2012) and Larocque et al. (2017) who examined the neural dissociation of items in working memory by pairing a retro-cueing method, similar to that used in our investigation, with a concurrent fMRI. These studies found that during the delay period that followed a retro-cue, the neural activity associated with the current-target (i.e., active) item remained constant while the activation associated with the now accessory representation, being held for later on, was reduced to baseline levels. The authors provided numerous possible alternatives to explain the decrease in accessory-associated activation following the retro-cue. These included the possibility that the representation of the accessory item may be maintained during the delay period via an increase in synaptic connections rather than electrical impulses. While determining the exact cause of the decrease in accessory-associated activation is beyond the scope of this current manuscript, the finding that accessory-associated activation is reduced to baseline levels but no further is consistent with the many cognitive-behavioural findings suggesting that the role of inhibition in this paradigm is not to facilitate search, but instead to limit accessory-associated activation until the accessory item is needed later on.

Our finding that currently irrelevant items undergo inhibition that reduces their influence during search aligns with another well-known cognitive-behavioural paradigm, preview search. Fenske et al. (2004) found that the inhibition underlying preview search 
is accompanied by a devaluation of previewed distractors. The devaluation in our results that takes place following the presentation of a retro-cue implies that the inhibition that caused the devaluation would have to have been applied directly to representations in memory. This differs from Fenske et al.'s findings regarding devaluation in preview search but yields a similar result, that is, devaluation of the task-irrelevant stimuli. It seems as though a similar mechanism causes devaluation for both externally present stimuli as well as those present at the interface between attention and working memory. In a past investigation, we found that using inhibitory processes to ignore an item that matched a feature of our current attentional template is accompanied by affective consequences (De Vito, Al-Aidroos, \& Fenske, 2017). Participants held a cued item in working memory and later were asked to choose the original from an array containing the target and a rotated version of the target. During the retention interval between memorization and test, a distractor array was presented that included one item that matched a feature (i.e., colour) of the target and another that did not. Participants not only inhibited the target-matching item, as evidenced by the elicitation of a Pd component, but also affectively devalued memory-matching items. This finding is evidence that memorymatching items that are currently irrelevant to our goals are inhibited and devalued when they are presented in our environment. Pairing these findings with our investigation of devaluation in working memory (De Vito et al., 2018) and the results reported here supports the suggestions that working memory is "internal attention" (Anderson \& Spellman, 1995; Chun, Golomb, \& Turk-Browne, 2011). The link between attentional and emotional processes that governs the prioritization of stimuli in our external 
environment seems to work very similarly during the prioritization of representations held solely in working memory.

\begin{abstract}
Author Note
This research was supported by the Natural Science and Engineering Research Council of Canada, the Canada Foundation for Innovation, and the Ontario Ministry of Research and Innovation. Correspondence concerning this article should be addressed to Mark J. Fenske, Ph.D., Department of Psychology, University of Guelph, Guelph, Ontario, Canada, N1G 2W1. Email: mfenske@uoguelph.ca. Our thanks to Michaela Comrie for her help with data collection and to Gavin Petrie for his help with earlier investigations of these concepts.
\end{abstract}




\section{References}

Anderson, M. C., \& Spellman, B. A. (1995). On the status of inhibitory mechanisms in cognition: memory retrieval as a model case. Psychological Review, 102(1), 68-100.

Carlisle, N. B., \& Woodman, G. F. (2011). Automatic and strategic effects in the guidance of attention by working memory representations. Acta Psychologica, 137(2), 217-225. http://doi.org/10.1016/j.actpsy.2010.06.012

Chetverikov, A. (2014). Warmth of familiarity and chill of error: Affective consequences of recognition decisions. Cognition and Emotion, 28(3), 385-415. http://doi.org/10.1080/02699931.2013.833085

Chetverikov, A., Jóhannesson, O. I., \& Kristjánsson, A. (2015). Blaming the victims of your own mistakes: How visual search accuracy influences evaluation of stimuli. Cognition and Emotion, 29(6), 1091-1106.

Chun, M. M., Golomb, J. D., \& Turk-Browne, N. B. (2011). A taxonomy of external and internal attention. Annual Review of Psychology, 62, 73-101. http://doi.org/10.1146/annurev.psych.093008.100427

Craver-Lemley, C., \& Bornstein, R. F. (2006). Self-generated visual imagery alters the mere exposure effect. Psychonomic Bulletin \& Review, 13(6), 1056-1060. http://doi.org/10.3758/BF03213925

De Vito, D., Al-Aidroos, N., \& Fenske, M. J. (2017). Neural evidence that inhibition is linked to the affective devaluation of distractors that match the contents of working memory. Neuropsychologia, 99, 259-269. http://doi.org/10.1016/j.neuropsychologia.2017.03.022

De Vito, D., \& Fenske, M. J. (2017). Suppressing memories of words and familiar objects results in their affective devaluation: Evidence from Think/No-think tasks. Cognition, COGNIT-D-1, 1-37. http://doi.org/10.1016/j.cognition.2017.01.020

De Vito, D., Ferrey, A. E., Fenske, M. J., \& Al-Aidroos, N. (2018). Cognitivebehavioural and electrophysiological evidence of the affective consequences of ignoring stimulus representations in working memory. http://doi.org/10.17605/OSF.IO/5S4N9

Desimone, R., \& Duncan, J. (1995). Neural Mechanisms of Selective Visual Attention. Annual Review of Neuroscience, 18(1), 193-222. http://doi.org/10.1146/annurev.neuro.18.1.193

Downing, P. E., \& Dodds, C. M. (2004). Competition in visual working memory for control of search. Visual Cognition, 11(6), 689-703. http://doi.org/10.1080/13506280344000446

Eimer, M. (2015). Visual working memory and attentional object selection. In P. Jolicœur, C. Lefebvre, \& J. Martinez-Trujillo (Eds.), Mechanisms of sensory working memory, Attention and Performance XXV (pp. 90-104). Amsterdam: Elsevier.

Faul, F., Erdfelder, E., Lang, A.-G., \& Buchner, A. (2007). GPOWER: A general power analysis program. Behavior Research Methods, 39(2), 175-191. http://doi.org/10.3758/BF03193146

Fenske, M. J., \& Raymond, J. E. (2006). Affective influences of selective attention. Current Directions in Psychological Science, 15(6), 312-316. http://doi.org/10.1111/j.1467-8721.2006.00459.x 
Fenske, M. J., Raymond, J. E., Kessler, K., Westoby, N., \& Tipper, S. P. (2005). Attentional inhibition has social-emotional consequences for unfamiliar faces. Psychological Science, 16(10), 753-758. http://doi.org/10.1111/j.14679280.2005.01609.x

Fenske, M. J., Raymond, J. E., \& Kunar, M. a. (2004). The affective consequences of visual attention in preview search. Psychonomic Bulletin \& Review, 11(6), 10551061. http://doi.org/10.3758/BF03196736

Ferrey, A. E., Frischen, A., \& Fenske, M. J. (2012). Hot or not: response inhibition reduces the hedonic value and motivational incentive of sexual stimuli. Frontiers in Psychology, 3(December), 575. http://doi.org/10.3389/fpsyg.2012.00575

Gollwitzer, P. M., Martiny-Huenger, T., \& Oettingen, G. (2014). Affective Consequences of Intentional Action Control. In Advances in Motivation Science (Vol. 1, pp. 4983). http://doi.org/10.1016/bs.adms.2014.08.002

Henson, R. N. A., \& Rugg, M. D. (2003). Neural response suppression, haemodynamic repetition effects, and behavioural priming. Neuropsychologia, 41(3), 263-270. http://doi.org/10.1016/S0028-3932(02)00159-8

Houtkamp, R., \& Roelfsema, P. R. (2006). The effect of items in working memory on the deployment of attention and the eyes during visual search. Journal of Experimental Psychology. Human Perception and Performance, 32(2), 423-442. http://doi.org/10.1037/0096-1523.32.2.423

Inoue, K., Yagi, Y., \& Sato, N. (2018). The mere exposure effect for visual image. Memory \& Cognition, 46(2), 181-190. http://doi.org/10.3758/s13421-017-0756-6

Kihara, K., Yagi, Y., Takeda, Y., \& Kawahara, J. I. (2011). Distractor Devaluation Effect in the Attentional Blink: Direct Evidence for Distractor Inhibition. Journal of Experimental Psychology: Human Perception and Performance, 37(1), 168-179. http://doi.org/10.1037/a0019948

Kirby, T. A., \& Greenwald, A. G. (2017). Mental ownership: Does mental rehearsal transform novel stimuli into mental possessions? Journal of Experimental Social Psychology, 73, 125-135. http://doi.org/10.1016/j.jesp.2017.05.004

Kiss, M., Raymond, J. E., Westoby, N., Nobre, A. C., \& Eimer, M. (2008). Response inhibition is linked to emotional devaluation: behavioural and electrophysiological evidence. Frontiers in Human Neuroscience, 2(October), 13. http://doi.org/10.3389/neuro.09.013.2008

Kornblith, S., Quian Quiroga, R., Koch, C., Fried, I., \& Mormann, F. (2017). Persistent Single-Neuron Activity during Working Memory in the Human Medial Temporal Lobe. Current Biology, 27(7), 1026-1032. http://doi.org/10.1016/j.cub.2017.02.013

Larocque, J. J., Riggall, A. C., Emrich, S. M., \& Postle, B. R. (2017). Within-Category Decoding of Information in Different Attentional States in Short-Term Memory. Cerebral Cortex, 27(10), 4881-4890. http://doi.org/10.1093/cercor/bhw283

Lewis-Peacock, J. A., Drysdale, A. T., Oberauer, K., \& Postle, B. R. (2012). Neural Evidence for a Distinction between Short-term Memory and the Focus of Attention. Journal of Cognitive Neuroscience, 24(1), 61-79. http://doi.org/10.1162/jocn_a_00140

Martiny-Huenger, T., Gollwitzer, $\bar{P}$., \& Oettingen, G. (2014). Distractor Devaluation in a Flanker Task: Object-Specific Effects Without Distractor Recognition Memory. Journal of Experimental Psychology: Human Perception and Performance, 40(2), 
613-625. Retrieved from

http://psycnet.apa.org.subzero.lib.uoguelph.ca/journals/xhp/40/2/613.pdf

Nunoi, M., \& Yoshikawa, S. (2016). Deep processing makes stimuli more preferable over long durations. Journal of Cognitive Psychology, 28(6), 756-763.

http://doi.org/10.1080/20445911.2016.1189917

Olivers, C. N. L., \& Meeter, M. (2008). A boost and bounce theory of temporal attention. Psychological Review, 115(4), 836-863. http://doi.org/10.1037/a0013395

Olivers, C. N. L., Peters, J., Houtkamp, R., \& Roelfsema, P. R. (2011). Different states in visual working memory: when it guides attention and when it does not. Trends in Cognitive Sciences, 15(7), 327-34. http://doi.org/10.1016/j.tics.2011.05.004

Peirce, J. W. (2007). PsychoPy-Psychophysics software in Python. Journal of Neuroscience Methods, 162(1-2), 8-13. http://doi.org/10.1016/j.jneumeth.2006.11.017

Peters, J. C., Goebel, R., \& Roelfsema, P. R. (2009). Remembered but Unused: The Accessory Items in Working Memory that Do Not Guide Attention. Journal of Cognitive Neuroscience, 21(6), 1081-1091. http://doi.org/10.1162/jocn.2009.21083

Peters, J. C., Roelfsema, P. R., \& Goebel, R. (2012). Task-Relevant and Accessory Items in Working Memory Have Opposite Effects on Activity in Extrastriate Cortex. Journal of Neuroscience, 32(47), 17003-17011. http://doi.org/10.1523/JNEUROSCI.0591-12.2012

Raymond, J. E. (2009). Interactions of attention, emotion and motivation. Progress in Brain Research, 176, 293-308.

Raymond, J. E., Fenske, M. J., \& Tavassoli, N. T. (2003). Selective attention determines emotional responses to novel visual stimuli. Psychological Science, 14(6), 537-42. Retrieved from http://www.ncbi.nlm.nih.gov/pubmed/14629683

Raymond, J. E., Fenske, M. J., \& Westoby, N. (2005). Emotional devaluation of distracting patterns and faces: a consequence of attentional inhibition during visual search? Journal of Experimental Psychology. Human Perception and Performance, 31(6), 1404-1415. http://doi.org/10.1037/0096-1523.31.6.1404

Raymond, J. E., Shapiro, K. L., \& Arnell, K. M. (1992). Temporary Suppression of Visual Processing in an RSVP Task: An Attentional Blink? Journal of Experimental Psychology: Human Perception and Performance, 18(3), 849-860.

Reeder, R. R., Olivers, C. N. L., \& Pollmann, S. (2017). Cortical evidence for negative search templates. Visual Cognition, 25(1-3), 278-290. http://doi.org/10.1080/13506285.2017.1339755

Schacter, D. L., \& Buckner, R. L. (1998). Priming and the Brain. Neuron, 20, 185-195. Retrieved from http://www.ncbi.nlm.nih.gov/entrez/query.fcgi?db=pubmed\&cmd=Retrieve\&dopt= AbstractPlus\&list_uids=959311384869848337related:EcUnNJ8oUA0J

van Moorselaar, D., Olivers, C. N. L., Theeuwes, J., Lamme, V. A. F., \& Sligte, I. G. (2015). Forgotten But Not Gone: Retro-Cue Costs and Benefits in a Double-Cueing Paradigm Suggest Multiple States in Visual Short-Term Memory. Journal of Experimental Psychology: Learning, Memory, and Cognition, 41(6), 1755-1763. http://doi.org/10.1037/xlm0000124

van Moorselaar, D., Theeuwes, J., \& Olivers, C. N. (2014). In competition for the attentional template: can multiple items within visual working memory guide 
attention? Journal of Experimental Psychology. Human Perception and Performance, 40(4), 1450-1464. http://doi.org/10.1037/a0036229

van Moorselaar, D., Theeuwes, J., \& Olivers, C. N. L. (2016). Learning changes the attentional status of prospective memories. Psychonomic Bulletin \& Review, 14831490. http://doi.org/10.3758/s13423-016-1008-7

Vivas, A. B., Marful, A., Panagiotidou, D., \& Bajo, T. (2016). Instruction to forget lead to emotional devaluation. Cognition, 150, 85-91. http://doi.org/10.1016/j.cognition.2016.02.005

Vorberg, D., Mattler, U., Heinecke, A., Schmidt, T., \& Schwarzbach, J. (2003). Different time courses for visual perception and action priming. Proceedings of the National Academy of Sciences of the United States of America, 100(10), 6275-80. http://doi.org/10.1073/pnas.0931489100

Watson, D. G., \& Humphreys, G. W. (1997). Visual Marking: Prioritizing Selection for New Objects by Top-Down Attentional Inhibition of Old Objects. Psychological Review, 104(1), 90-122.

Watson, D. G., \& Humphreys, G. W. (2000). Visual marking: evidence for inhibition using a probe-dot detection paradigm. Perception \& Psychophysics, 62(3), 471-481. http://doi.org/10.3758/BF03212099

Wessel, J., \& O’Doherty, J. (2014). Stimulus devaluation induced by stopping action. Journal of Experimental Psychology: General, 143(6), 2316-2329. Retrieved from http://psycnet.apa.org/journals/xge/143/6/2316/

Wolfe, J. M. (1994). Guided Search 2 . 0 A revised model of visual search. Psychnomic Bulletin \& Review, 1(2), 202-238. http://doi.org/10.3758/BF03200774

Woodman, G. F., \& Luck, S. J. (2007). Do the Contents of Visual Working Memory Automatically Influence Attentional Selection During Visual Search? Journal of Experimental Psychology. Human Perception and Performance, 33(2), 363-377. http://doi.org/10.1007/s11103-011-9767-z.Plastid

Yagi, Y., Ikoma, S., \& Kikuchi, T. (2009). Attentional modulation of the mere exposure effect. Journal of Experimental Psychology. Learning, Memory, and Cognition, 35(6), 1403-1410. http://doi.org/10.1037/a0017396

Zago, L., Fenske, M. J., Aminoff, E., \& Bar, M. (2005). The rise and fall of priming: How visual exposure shapes cortical representations of objects. Cerebral Cortex, 15(11), 1655-1665. http://doi.org/10.1093/cercor/bhi060

Zajonc, R. B. (1968). Attitudinal effects of mere exposure. Journal of Personality and Social Psychology, 9(2).

Zajonc, R. B. (2001). Mere exposure: A gateway to the subliminal. Current Directions in Psychological Science, 10(6), 224-228. http://doi.org/10.1111/1467-8721.00154 


\begin{tabular}{lll}
\hline & Group & \\
\cline { 2 - 3 } Number of trials & No-search & Search \\
\hline Baseline & 64 & 32 \\
Critical & 64 & 32 \\
Search & - & 72 \\
Event durations (ms) & & \\
\hline Word cues & 500 & 800 \\
Memory array & 500 & 500 \\
Retention intervals & 800 & 1000 \\
Retro-cues & 200 & 200 \\
Memory probes & 1000 & 1000 \\
Search array & - & till response \\
\hline
\end{tabular}

Table 1. Number of trials and event durations (ms) for the No Search and Search groups. 


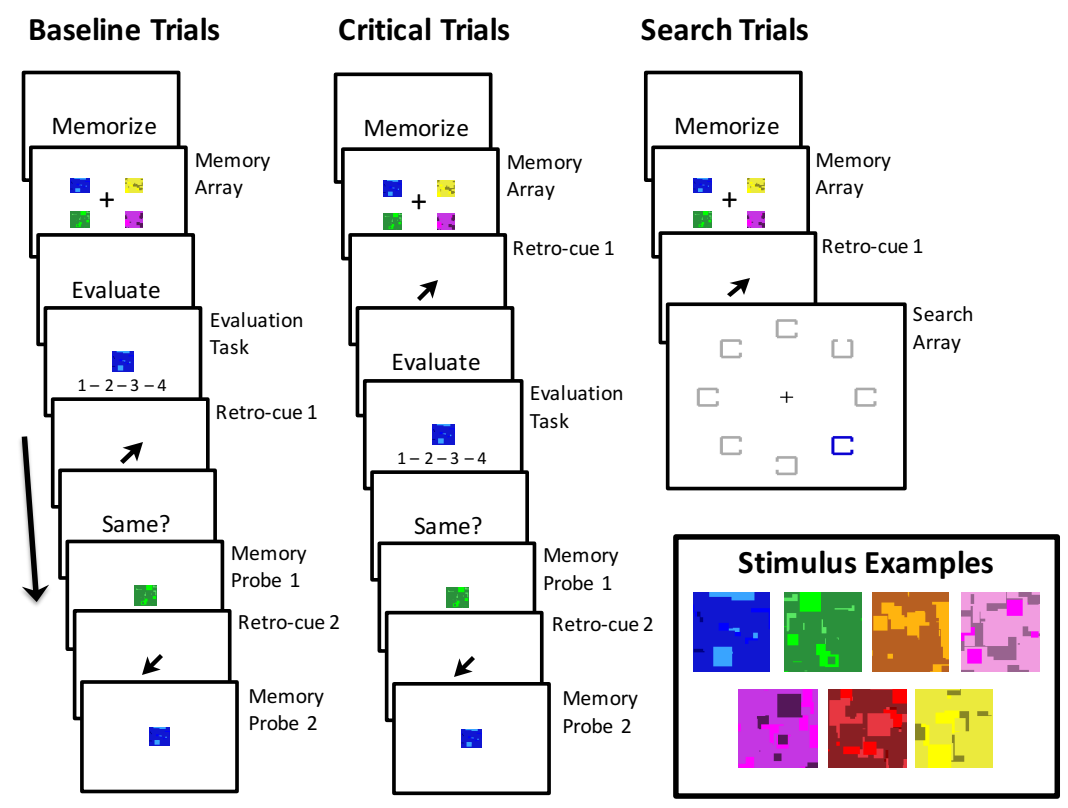

Figure 1. Stimulus examples and sequence of trials. While participants in the No Search group only completed Baseline Trials and Critical Trials, participants in the Search group also completed Visual-search trials. 


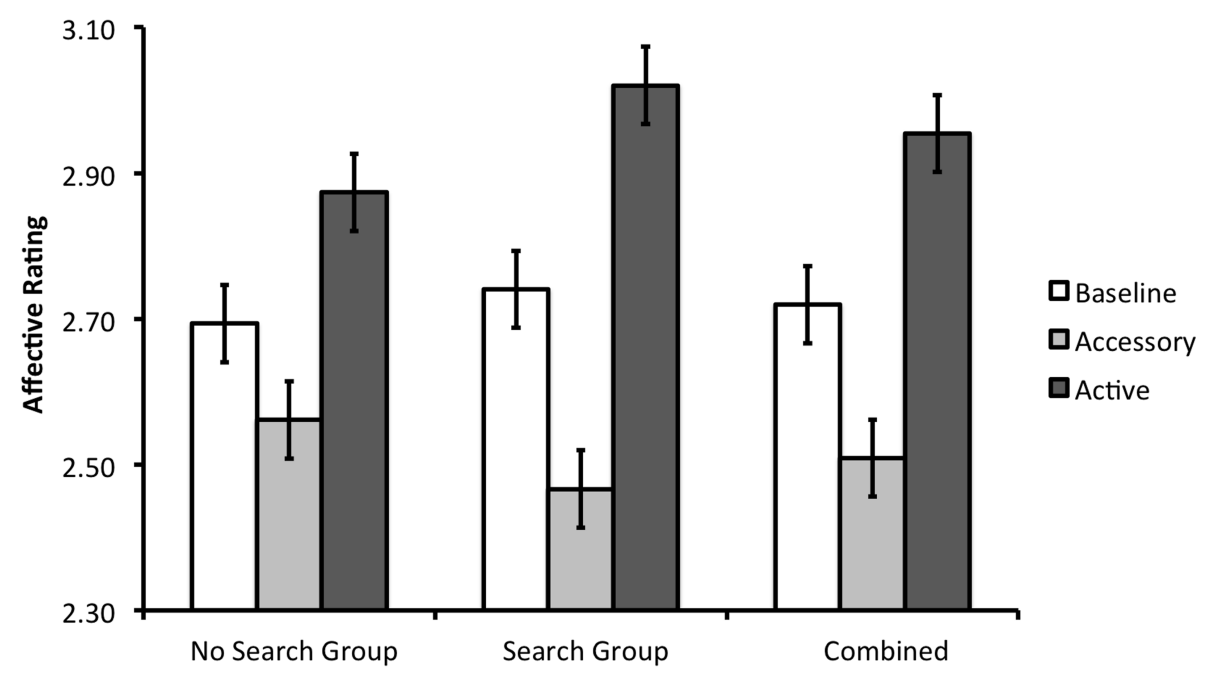

Figure 2. Affective Ratings for Baseline, Accessory, and Active items. Error bars represent within-subjects standard errors of the means.

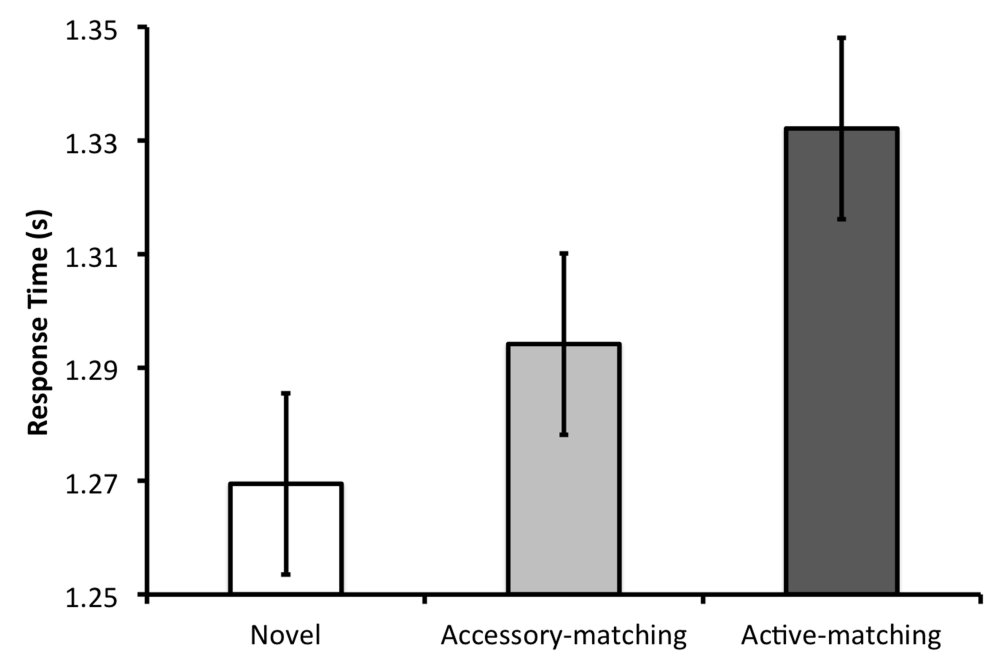

Figure 3. Response times on search task trials for participants in the Search Group. Error bars represent within-subjects standard errors of the means. 